\title{
Umbilical Cord Prolapse and Management Upon Medical Personnel Skills - Case Report
}

\author{
Rustem Celami $^{1 *} \quad$ Spiro Damo $^{2} \quad$ Niketa Koliçi $^{3} \quad$ Erjon Panajoti $^{3}$ \\ 1. Faculty of Medical Technical Sciences, University of Aldent and American Hospital of Tirana, Albania. \\ Albania \\ 2. Queen Geraldine University Hospital of Obstetrics and Gynecology, Tirana, Albania \\ 3. American Hospital of Tirana, Tirana, Albania \\ *Corresponding author Rustem Celami, MD, PhD, Associate Professor
}

\begin{abstract}
Umbilical cord prolapse is obstetric emergency that may have significant neonatal morbidity and mortality if not prompt professional management does not take place. Umbilical cord prolapse is the descended umbilical cord via collum uteri with proceeding presenting part after ruptured of membranes of the amniotic fluid.

Case Report

A 31 years old pregnant woman, comes to maternity service after her waters were broken (ruptured membranes of amniotic fluid), spontaneously at home. It was her second pregnancy; her first pregnancy was vaginal delivery 3 years ago. Actual pregnancy according to her last menstrual period was term pregnancy; she stated that was followed up during pregnancy by her obstetrician. Within very few minutes, patient was seen by admission midwife, where vaginal examination was performed and umbilical cord prolapsed was identified. The fetal heart rate was listened by Pinard horn obstetrical stethoscope and bradyarrythmia was present. The on duty obstetrician was called, while patient was kept on obstetrical lithotomy position by midwife. Bimanual pelvic examination was performed by obstetrician on duty, the prolapsed of umbilical cord was confirmed and bradyarrythmia of fetal heart was present. At this time, patient was taken to operating room, and electronic fetal monitoring was performed, where fetal bradyarrythmia $90 \mathrm{FHR} / \mathrm{min}$ and immediate Cesarean section delivery was performed, baby did cry immediately after delivery, Apgar score 8/9 referred neonatologist assessment.

Conclusion, umbilical cord prolapse is a life threatening event for the fetus and requires immediate intervention. Medical personnel should be well trained to address such emergency proper and professional management.

Keywords: Umbilical cord prolapse, obstetrical emergency, mortality, morbidity, fetus

DOI: $10.7176 /$ ALST/80-04

Publication date: April $30^{\text {th }} 2020$

\section{Introduction}

Umbilical cord prolapse is a life threatening obstetrical emergency for the fetus and requires immediate intervention. The cord slips ahead of presenting part of fetus and protrudes into cervical canal or vagina, or outside in the introitus. It is an obstetrical emergency because the prolapsed cord is vulnerable to compression, umbilical vein occlusion, and umbilical artery vasospasm, which can compromise fetal oxygenation. Umbilical cord prolapse can occur prior to or during delivery of the fetus, but it often occurs when the membranes rupture (ROM). The prolapse of umbilical cord is an uncommon obstetric emergency but with potentially significant neonatal adverse outcomes. The incidence of umbilical cord prolapse according to recent studies is reported to be $0.1 \%-0.6 \%[1,2,4]$. Such obstetrical emergency cases of umbilical cord prolapse appear often and many studies have concluded with a perinatal mortality rate of over $90 / 1000[1,2,3,4]$.

Umbilical cord prolapsed, illustrated in figure 1, chiefly occurs; primarily, when presenting part does not sufficiently fill the pelvis because of maternal or fetal characteristics, and secondly, after obstetric interventions which displace the presenting part. While many observational studies suggest that obstetric interventions increase risk of umbilical cord prolapse, it is often difficult to determine whether umbilical cord prolapse would have occurred spontaneously if the intervention had not been performed $[5,7,9,12]$.
\end{abstract}



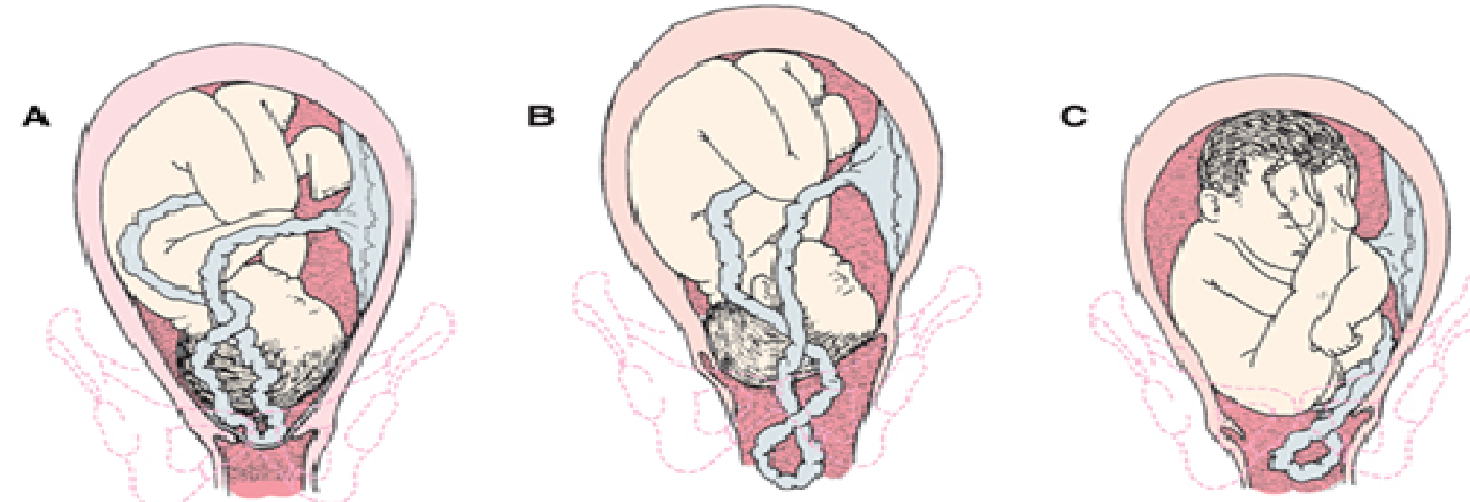

Figure 1. Illustration of prolapsed of umbilical cord. A - Complete occult prolapsed; B - Cord presenting in front of the fetal head; may be seen in the vagina; $\mathrm{C}$ - Frank breech presentation with prolapsed cord

\section{Discussion}

The prolapsed of umbilical cord usually presents with severe and prolonged fetal bradycardia, presence of late deceleration on fetal electronic monitoring, and on vaginal examination is felt by finger/s the umbilical cord. In Albania, as in many countries pelvic bimanual examination is performed by both obstetrician and midwife. As risk or predisposing factors, mentioning a few; amniotomy before presenting part descent and engagement, premature rupture of membranes, fetal malpresentation like breech or transverse, premature rupture of membranes, small babies, multiple pregnancies, polyhydramnios, disproportion cephalopelvic $[1,5,6,12]$. Medical interventions may predispose umbilical cord prolapsed as well, mentioning a few; artificial rupture of membranes, especially when this is done not in lithotomy position of obstetrical - gynecological bimanual pelvic exam table, internal scalp electrode application, intrauterine pressure catheter insertion, manual internal rotation, amnioinfusion or amnioreduction, application ofr foreceps or vaccum $[1,5,6,10,11]$.

Compression of umbilical cord leads to either profound/total acute asphyxia or subacute hypoxia with different neonatal outcomes. It has been suggested that the pathological mechanism of umbilical cord prolapse is almost accompanied medical fetal consequence from severe neurological damage and even death or causing little or no cerebral injury and this is supported by very low incidence of stillbirth/neonatal death, neonatal encephalopathy, and cerebral palsy $[1,6,7,8,9,12]$.

Rupture of membranes take place either iatrogenic when medical professionals rupture amniotic membranes in synergy with labor stage, or can be spontaneously preterm or when vaginal infection is present or other pathological factors, and it also can happen when labor activity begins.

This pregnant lady comes to the maternity service of a regional hospital, she was 31 years old, and it was her second pregnancy, and her first pregnancy was delivered in term by vaginal route, with no medical problem to mention. At current visit, patient stated that the waters broke a few minutes ago at her house, and she came immediately to the hospital. Obstetrical admission room, located on $3^{\text {rd }}$ floor of maternity, where patient was assessed by admission midwife, where a vaginal examination was performed and prolapse umbilical cord was noted. Fetal Heart Rates were assessed by Pinard horn obstetrical stethoscope and bradyarrythmia was present, $80-90 \mathrm{FHR} / \mathrm{min}$, electronic fetal monitoring not available at our obstetrical admission room. The on duty obstetrician was called, while patient was kept on obstetrical lithotomy position by midwife. However, the obstetrician noticed that midwife hand was not into the vagina, even though was told that umbilical cord prolapse was evident. Bimanual pelvic examination was performed by obstetrician on duty immediately, the prolapsed of umbilical cord was confirmed and bradyarrythmia of fetal heart was present. Using two fingers, a maneuver, making a spiral circle, to slide umbilical cord inside that was tighten to fetal head was done at the moment.

At this time, patient was taken on wheelchair, heading toward the elevator, that unfortunately were not in service, then patients was taken through stairs, while on the wheelchair, with the help of medical staff and a couple of patient family members. Patients was taken to the ground floor to obstetrical ward that was next to operating room, and electronic fetal monitoring was performed, where fetal bradyarrythmia $90 \mathrm{FHR} / \mathrm{min}$. Once again, another immediate vaginal examination was performed, umbilical cord in vagina was felt again, and the fetal head was pushed up by obstetrician hand, while urgent operating room was ordered and ready. Patient was taken to operating room, intubated by anesthesiologist on duty immediately; a quick abdominal antiseptic preparation was performed at incision site. The physician examination hand on patient's vagina pushing fetal head was replaced by midwife's hand, and immediate Cesarean section delivery was performed, baby did cry immediately after delivery and was with Apgar score 8/9 referred neonatologist assessment. The time measured from first visit to delivery was 6-7 minutes. 
During this emergency, few problems were faced. First of all, the midwife with many years' experience did take off her hand from vaginal examination; even she did notice the umbilical cord prolapse, making the fetal health vulnerable to hypoxia and even with severe or fatal consequences if not timely management was not offered. We believe, this non appropriate approach happens generally because, of not solid knowledge and skills of obstetrical emergencies of medical staff. Some emergencies do not happen that often and also there is no such emergency simulations performed on our hospital setting so the medical staff can have a brief of such emergencies and improve their knowledge and approachable skills as well. The other thing that was noticed was that in the admission room, due to lack of infrastructure we do not have electronic fetal monitoring, because using only classic means such as using only Pinard stethoscope may create delays of accurate assessment of fetal heart arrhythmias, especially of young or not well experiences medical staff.

In addition, there is absolutely out of any logic and beyond any hospital architecture model, the location of obstetrical admission room far away from obstetrical delivery room or operating room, like in this case admission office of obstetrics located on the third floor of the hospital, when labor ward and operating rooms are located on the ground floor. The other problem was the elevator, of course a non-working elevator and being third floor, respectively making a considerable difference length \route from admission to operation room contributing to delays and time wasting and in such case, that may have severe fetal consequences to fetal health. In conclusion, umbilical cord prolapse is a life threatening event for the fetus and requires immediate intervention. Medical staff must be trained and re-trained periodically in order to keep their awareness and skills up to date, and emergency medical stimulations are evidence based training that helps to improve the response. Physical infrastructure and medical equipments play another important role beyond the human resources availability and ability, and lack of these should be addressed professionally and institutionally. These problems are presented also to respective responsible professional institutions and hopefully will be addressed properly, in order to overall improve medical service in our country.

\section{References}

1. Gibbons C, O'Herlihy C, Murphy JF. Umbilical cord prolapse--changing patterns and improved outcomes: a retrospective cohort study. BJOG 2014; 121:1705.

2. Myles TJ. Prolapse of the umbilical cord. J Obstet Gynaecol Br Emp 1959;66:301-10.

3. Kahana B, Sheiner E, Levy A, Lazer S, Mazor M. Umbilical cord prolapse and perinatal outcomes. Int J Gynaecol Obstet 2004;84:127-32.

4. Critchlow CW, Leet TL, Benedetti TJ, Daling JR. Risk factors and infant outcomes associated with umbilical cord prolapse: a population-based case-control study among births in Washington State. Am J Obstet Gynecol 1994;170:613-8.

5. Royal College of Obstetricians and Gynecologists . Umbilical Cord Prolapse: Green-top Guideline No. 50. London, UK: RCOG; 2014.

6. Ylä-Outinen A, Heinonen PK, Tuimala R. Predisposing and risk factors of umbilical cord prolapse. Acta Obstet Gynecol Scand 1985;64:567-70.

7. Woo JS, Ngan YS, Ma HK. Prolapse and presentation of the umbilical cord. Aust N Z J Obstet Gynaecol 1983;23:142-5.

8. Panter KR, Hannah ME. Umbilical cord prolapse: so far so good? Lancet 1996;347:74.

9. Faiz SA, Habib FA, Sporrong BG, Khalil NA. Results of delivery in umbilical cord prolapse. Saudi Med J 2003;24:754-7.

10. Centre for Maternal and Child Enquiries (CMACE). Perinatal Mortality 2008: United Kingdom. London: CMACE; 2010

11. Maternal and Child Health Research Consortium. Confidential Enquiry into Stillbirths and Deaths in Infancy. 7th Annual Report Focusing on Breech Presentation at Onset of Labour, Obstetric Anaesthesia Delays and Complications, Cardiotocograph Education Survey and Sudden Unexpected Deaths in Infancy Pathology. London: Maternal and Child Health Research Consortium; 2000.

12. Confidential Enquiry into Maternal and Child Health (CEMACH). Perinatal Mortality 2006: England, Wales and Northern Ireland. London: CEMACH; 2008 